\title{
THE CIRCULATING BLOOD VOLUME OF PARA- AND TETRAPLEGICS IN CORRELATION TO SERUM FACTORS
}

\author{
By G. Lob, J. Seifert, E. Stoephasius and J. Probst \\ Institute for Surgical Research of the University of Munich and \\ Unfallkrankenhaus Murnau Abstract
}

THE knowledge of the circulating blood volume is of high therapeutical value not only in haemorrhagic shock but also in shock due to spinal injuries. To get reliable values of the blood volume in para- and tetraplegics we studied the blood volume using two different methods and with respect to parameters which possibly influence blood volume values.

\section{METHODS}

Fifty para- or tetraplegic patients received either ${ }^{51} \mathrm{Cr}$-labelled erythrocytes or $\mathrm{R}^{125}$ IHSA intravenously. After a period of $\mathrm{I}, 5, \mathrm{IO}, 20,30$ and 60 minutes I ml. blood was withdrawn from the contralateral vein. The radio-activity was measured in a well-type scintillation counter and the mixing time, elimination rate and the circulating blood volume were calculated. Furthermore, hemmatocrit, protein content and colloid osmotic pressure was determined in every sample. Similar investigations were made in a control group of 20 healthy volunteers.

\section{RESULTS}

Blood volume is markedly influenced by the complete mixing and elimination of the blood volume indicator. Both parameters are changed in spinal cord injuries. Whereas in normal men both tracers are completely mixed within 8 minutes. In spinal cord injuries this mixing time is prolonged for up to 22 minutes, this climation of ${ }^{51} \mathrm{Cr}$-labelled erythrocytes remains unaffected in para- and tetraplegics. But the disappearance rate of $\mathrm{R}^{125} \mathrm{IHSA}$ is increased from 8 per cent. per hour in controls to I5 per cent. per hour in patients. All other parameters were not significantly changed. With regard to the prolonged mixing time and the elevated disappearance rate blood volume values were similar to blood volume found in normal human beings.

\section{CONCLUSION}

Measuring blood volume in patients with spinal cord injuries a changed mixing time of the tracer and an increased disappearance of $\mathrm{R}^{125}$ IHSA must be taken into consideration. Exact datas can be calculated only with ${ }^{51} \mathrm{Cr}$-labelled erythrocytes because the elimination of $\mathrm{R}^{125}$ IHSA renders the calculation of circulating blood volume more difficult. 